\title{
A DISTRIBUIÇĀO DE PROBABILIDADE DOS RETORNOS DAS AÇÕES NO BRASIL: UMA ABORDAGEM NĀO-PARAMÉTRICA
}

\author{
Sylvia Delgado*
}

\section{Resumo}

Nesse artigo estimamos não-paramétricamente as distribuições de probabilidade dos retornos das ações das empresas Petrobrás, Eletrobrás, Paranapanema e Telebrás, usando o estimador de densidade conhecido como estimador de núcleo. Para este último, apresentamos os resultados estabelecidos para amostras independentes e um teorema central funcional do limite para amostras dependentes, com o qual estimamos os intervalos de confiança das densidades estimadas. Esse teorema é necessário no nosso caso porque os retornos de ações não podem ser considerados independentes.

Nosso objetivo consiste essencialmente na comparação entre as densidades estimadas e distribuição Normal. Procuramos verificar, em particular, a existência de assimetria, leptocurtose e caudas pesadas nas distribuições estimadas. Estas três caracteristicas são recorrentes nas distribuições de probabilidade dos retornos de ativos financeiros e implicam em não-normalidade. Concluimos em todos os casos que não se pode aceitar a normalidade dos retornos dessas ações. Essa conclusão é confirmada pelos testes de normalidade que efetuamos sobre esses retornos.

* Escola de Pós Graduação em Economia, Fundação Getúlio Vargas. Agradeço Flôres e Sérgio R da Costa Wérlang e os comentários e conselhas de João Victor Issler. Agradeço igualrnente esse trabalho. Os erros remanescentes são de minha inteira responsabilidade.

R. de Econometria Rio de Janeiro v.15, $\mathrm{n}^{\mathrm{O}}$ 1, pp.77-100 Abril/Outubro 1995 


\begin{abstract}
In this paper we estimate the probability distribution function of the daily return on stock of four Brazilian companies, using a non-parametric method, namely, the kernel estimator. Besides, as a comparison, we have performed three normality tests: Kolmogorov-Smirnov, Chi-Square and Jarque-Bera. We have concluded that the density function of the return of Eletrobras, Petrobras, Paranapanema and Telebras cannot be considered normally distributed.
\end{abstract}

Palcuras-Ch ve: Estimação não-paramétrica de densidade, estimador de núcleo, distribuição de probabilidade dos retornos de ações

Código JEL: C14, G12

\title{
1. Introdução.
}

A especificação da distribuição dos retornos das ações é fundamental para a tomada de decisões de quem investe nesses ativos financeiros: desde os modelos de carteira aos modelos de opções, os critérios de decisão são baseados nas distribuições de probabilidade. Por exemplo, um dos resultados mais importantes da teoria de seleção de carteira é que a utilidade esperada de um investimento varia diretamente com o primeiro momento da distribuição dos retornos do investimento e inversamente com o segundo, desde que satisfeitas 3 hipóteses básicas: (1) as preferências dos indivíduos podem ser representadas por uma função utilidade contínua, não-decrescente e estritamente cõncava; (2) os indivíduos maximizam sua utilidade esperada; e (3) os retornos são normalmente distribuídos.

A té o trabalho de Mandelbrot (1963), admitia-se que o processo gerador das variações de preço das ações era um movimento browniano, implicando por definição variância finita e distribuição normal com média $\mu$ e variância $\sigma^{2} t$. Mandelbrot, e mais tarde, Fama (1965) contestaram a hipótese de normalidade dos retornos baseando-se numa característica recorrente das distribuições empíricas: o excesso de observações nas caudas e em torno da média, quando comparadas 
com a distribuição normal. Isso os levou a sugerir que a densidade de probabilidade pertencia à classe das distribuições Pareto Estável com parâmetro $\alpha \in(0 ; 2]$. Quando $\alpha=2$, tem-se a distribuição normal, e no caso em que $\alpha<2$, a distribuição de probabilidade não possui variância finita, e é leptocúrtica.

O modelo de Mandelbrot foi considerado uma alternativa muito interessante ao modelo tradicional, apesar de supor a variância infinita, o que impossibilita o uso das mais importantes técnicas estatísticas. Nas décadas de 70 e 80 , a pesquisa sobre a distribuição de probabilidade de retornos seguiu dois rumos diferentes. Por um lado, procurou-se definir processos estocásticos capazes de gerar distribuições não-normais, e por outro lado, realizou-se um grande esforço no sentido de: (1) desenvolver métodos de estimação dos parâmetros da Pareto e (2) determinar qual dos três modelos - $t$ de Student, Normal ou Pareto $\operatorname{com} \alpha<2$ - se adequava melhor aos retornos.

Uma característica comum a todos esses trabalhos é a suposição de que a distribuição de retornos pertence a uma família paramétrica de distribuições, da qual estima-se os parâmetros. Esta é a abordagem convencional da estimação de densidade. Porém essa hipótese restritiva não é necessária, visto que existem métodos nãoparamétricos de estimação de densidade. De fato, a estimação nãoparamétrica não estabelece nenhuma estrutura formal para a função $f$ subjacente aos dados. Esta pode pertencer a uma família de distribuições muito ampla como, por exemplo, a família das densidades de probabilidade contínuas.

A estimação não-paramétrica revelou-se bastante precisa em análise exploratória de dados, que consiste na identificação das principais características de uma densidade: multimodalidade, curtose, assimetria, comportamento das caudas etc.

Esse tipo de análise é justamente o que queremos aplicar aos retornos de 4 ações brasileiras: Petrobrás, Eletrobrás, Telebrás e Paranapanema, adotando uma abordagem não-paramétrica, que nos parece 
mais adequada por não impor nenhuma restrição à densidade de probabilidade dos retornos dessas ações. Em particular, procuramos determinar se a distribuição de probabilidade desses retornos pode ser considerada uma Normal.

$\mathrm{Na}$ seção 2, apresentaremos brevemente o estimador de núcleo e suas principais propriedades. A seção 3 mostra os resultados da estimação de densidade das ações citadas e da comparação entre as distribuições estimadas e a Normal. A seção 4 contérn os resultados dos testes de normalidade e concluímos na seção 5 .

\section{Estimação Não-Paramétrica de Densidade.}

\subsection{Propriedades dos Estimadores.}

Um estimador $\hat{f}$ da função densidade de probabilidade é nãotendencioso se, para todo $x \in \Re, E[\hat{f}(x)]=f(x)$. Rosenblatt (1956) provou que nenhum estimador tal que $\hat{f}(x) \geq 0$ e $\int \hat{f}(x) d x=1$ é não-tendencioso para todas densidades contínuas, razão pela qual as pesquisas se concentram mais em seqüências $\left\{\hat{f}_{n}(x)\right\}$ de estimadores não-paramétricos que são assintoticamente não-viesados.

Se $\hat{f}_{n}(x) \rightarrow f(x)$ em probabilidade para todo $x \in \Re$ então $\hat{f}(x)$ é fracamente consistente e se a convergência for quase-certa, é fortemente consistente. Há quatro outros tipos de consistência, que dependem do critério do erro. Apresentamos um deles:

Se $f \in L_{2}$, i.e., $\int f(x)^{2} d x<\infty$, então o Erro Quadrático Médio Integrado de $\hat{f}(x)$ é definido por:

$$
E Q M I=\int_{-\infty}^{\infty} E_{f}[\hat{f}(x)-f(x)]^{2} d x
$$

No caso i.i.d., os parâmetros dos estimadores de densidade são escolhidos de forma ótima quando o EQMI é minimizado. 


\section{Sylvia Delgado}

\subsection{Estimador de Núcleo.}

Suponhamos uma amostra de tamanho $n, X_{1}, X_{2}, \cdots, X_{n}$ independentes e identicamente distribuídas como a variável aleatória $X$, cuja densidade $f$ deve ser estimada. Os estimadores serão designados por $\hat{f}(x)$.

O estimador de densidade mais popular depois do histograma é o chamado estimador de núcleo (kernel estimator). A idéia subjacente é a própria definição de densidade de probabilidade:

Se $X$ tem função de densidade de probabilidade $f$, então:

$$
\hat{f}(x)=\frac{1}{2 n h}\left[\mathrm{n} \text { o de } X_{i} \text { que pertencem a }(x-h ; x+h)\right] .
$$

Esse é conhecido como estimador ingênuo de $f$. Pode ser transformado num histograma fazendo-se de $x_{0}$ o centro do intervalo $T_{i}=\left[t_{n, i} ; t_{n, i+1}\right]$.

Uma maneira mais clara de exprimir esse estimador e que leva diretamente ao estimador de núcleo, é definir a função peso $w$ :

$$
w(x)=\left\{\begin{array}{lc}
\frac{1}{2} & |x|<1 \\
0 & \text { caso contrário }
\end{array}\right.
$$

O estimador ingênuo pode então ser reescrito como:

$$
\hat{f}(x)=\frac{1}{n} \sum_{i=1}^{n} \frac{1}{h} w\left(\frac{x-X_{i}}{h}\right) .
$$

Se $w(x)$ for uma função $K(x)$ satisfazendo $\int K(x) d x=1$, obtemos o estimador de núcleo:

$$
\hat{f}(x)=\frac{1}{n} \sum_{i=1}^{n} \frac{1}{h} K\left(\frac{x-X_{i}}{h}\right)
$$

Revista de Econometria 
onde $h$ é a amplitude da "janela" ou parâmetro de alisamento. $K(x)$ pode ser qualquer função, mas em geral, escolhe-se uma densidade de probabilidade para que $\hat{f}(x)$ também seja uma. Além disso, $f(x)$ herda as propriedades de continuidade e diferenciabilidade do núcleo $K$. Finalmente, observa-se que o estimador ingênuo pode ser considerado um estimador de núcleo.

O estimador de núcleo é fortemente consistente se $h \rightarrow 0$ e $n h \rightarrow \infty$ quando $n \rightarrow \infty$, independentemente de $f$; e Parzen (1962) mostrou que o estimador de núcleo univariado é assintoticamente não-tendencioso e normal se $h \rightarrow 0$ quando $n \rightarrow \infty$. A convergência de $E Q M I$ para 0 é da ordem de $n^{(-4 / d+4)}$ onde $d$ é a dimensão do núcleo, i.e., se o núcleo for univariado, $d$ é igual a 1.

\section{Escolha do Núcleo e da Amplitude Ótima.}

Há dois aspectos essenciais no estimador de núcleo: a função $I^{\prime}(x)$ e a amplitude $h$, da qual depende o resultado final da estimação. A escolha do núcleo $K(x)$ não tem maiores conseqüências, já que não influi sobre a consistência e a tendenciosidade do estimador; mas até pouco tempo, usava-se preferencialmente núcleos positivos e cuja integral era igual a um. Recentemente, mostrou-se que núcleos não necessariamente positivos podem melhorar a velocidade de convergência do EQMII (ver Gasser et al. (1985)).

O parâmetro de alisamento, $h$ é fundamental por dois motivos: a variância do estimador está basicamente relacionada ao número de pontos amostrais que pertencem ao intervalo $(x-h ; x+h)$, cuja amplitude é controlada por $h$; e por outro lado, há um trade-off entre o erro sistemático (viés) e o aleatório (variância) que depende de $h$. Mostraremos isso a seguir.

Seja $K(x)$ tal que $\int K(x) d x=1, \int t^{2} K(t)=k_{2} \neq 0 \mathrm{e}$ $\int t \Pi(t) d t=0 .^{1}$

1 Todas as integrais são de $-\infty$ a $+\infty$. 
O EQMI do estimador pode ser escrito como:

$$
\begin{aligned}
E Q M I & =\int E_{f}[\hat{f}(x)-f(x)]^{2} d x=\int V A R[\hat{f}(x)]+ \\
& +\{V I E S[\hat{f}(x)]\}^{2} d x .
\end{aligned}
$$

Estabelecendo-se $y=x-h t$ e expandindo $f(x-h t)$ numa série de Taylor até a segunda ordem, supondo-se $h$ pequeno e $n$ grande, obtém-se então o EQMI aproximado:

$$
\begin{aligned}
& E Q M I \approx \int \operatorname{VAR}[\hat{f}(x)] d x+\int\{\operatorname{VIES}[\hat{f}(x)]\}^{2} d x \\
& E Q M I \approx \frac{1}{n h} \int I K(t)^{2} d t+\frac{1}{4} h^{4} \int\left\{f^{\prime \prime}(x) k_{2}\right\}^{2} d x .
\end{aligned}
$$

Os detalhes da dedução desse resultado podem ser encontrados em Silverman (1986). Analisando-se (7) conclui-se que se escolhermos um valor muito pequeno para $h$, para eliminar o viés, a variância integrada torna-se muito grande; e escolhendo um $h$ alto, reduzir-seá a variância em detrimento do viés.

Nessas condiçø̃es, o valor ideal de $h$ é o que minimiza o $E Q M I$ como um todo. Parzen (1962) mostrou que a janela que minimiza (7), que é uma aproximação do $E Q M I$, é dada por:

$$
h^{*}=k_{2}^{-2 / 5}\left\{\int K(t)^{2} d t\right\}^{1 / 5}\left\{\int f^{\prime \prime}(x)^{2} d x\right\}^{-1 / 5} n^{-1 / 5}
$$

de onde se conclui que $h^{*} \rightarrow 0$ quando $n \rightarrow \infty$, mas a uma velocidade muito pequena: $O\left(n^{-1 / 5}\right)$. Além disso, essa janela ótima depende da função a ser estimada. Para se usar esse resultado, é preciso escolher $f(x)$ pertencente a alguma família de distribuições de probabilidade. A escolha natural é a normal $\Phi$ com variância $\sigma^{2}$, o que implica:

$$
\int f^{\prime \prime}(x)^{2} d x=\sigma^{-5} \int \Phi^{\prime \prime}(x)^{2} d x=\frac{3}{8} \pi^{-1 / 2} \sigma^{-5} \approx 0,212 \sigma^{-5}
$$


daí, $h^{*}=(4 \pi)^{-1 / 10} \frac{3}{8} \pi^{-1 / 2} \sigma n^{-1 / 5}$

$$
h^{*}=1,06 \sigma n^{-1 / 5}
$$

onde $\sigma$ pode ser substituído por $s$, o desvio-padrão amostral. $h^{*}$ dá bons resultados quando a densidade verdadeira é normal. Caso contrário, tende a alisar excessivamente (oversmooth) a densidade estimada, principalmente as multimodais. Uma janela robusta, $h^{*}=0,79 R n^{-1 / 5}$, onde $R$ é intervalo interquartílico, deve ser usada no lugar.

Em relação ao problema da escolha do núcleo, substituindo-se $h^{*}$ em (7), o Erro Quadrático Médio Integrado reduz-se a:

$$
\frac{5}{4} C(K)\left\{\int f^{\prime \prime}(x)^{2} d x\right\}^{1 / 5} n^{-4 / 5}
$$

onde

$$
C(K)=k_{2}^{2 / 5}\left\{\int K(t)^{2} d t\right\}^{4 / 5}
$$

De (10) deduz-se que, ceteris paribus, deve-se escolher um núcleo que minimize $C(K)$ - para poder minimizar o $E Q M I$ - e que esse problema significa minimizar:

$$
\int K(t)^{2} d t
$$

s.a. $\int t K(t) d t=1$ e $\int t^{2} K(t)=1$.

Hodges e Lehmann (1956) mostraram que o núcleo que é solução desse problema é:

$$
K_{e}(t)= \begin{cases}\frac{3}{4 \sqrt{5}}\left(1-\frac{1}{5} t^{2}\right) & -\sqrt{5} \leq t \leq \sqrt{5} \\ 0 \text { caso contrário } & \end{cases}
$$




\section{Sylvia Delgado}

o chamado núcleo de Epanechikov.

O critério de escolha do núcleo é sua eficiência, definida em relação ao núcleo de Epanechikov:

$$
e f f(K)=\left\{\frac{C\left(K_{e}\right)}{C(K)}\right\}^{5 / 4}
$$

Em Silverman (1986) encontram-se os valores das eficiências dos núcleos mais usados: gaussiano, biweight, uniforme, triangular. Verifica-e que a eficiência de todos estes núcleos varia entre 0,99 (biweight) e 0,92 (uniforme); a do gaussiano é igual a 0,95. Conclui-se então que o núcleo não interfere na estimação de densidade e que sua escolha deve obedecer a critérios práticos como a diferenciabilidade do núcleo ou a rapidez computacional da estimaşão.

A teoria exposta acima parte do pressuposto que a amostra é independente. Quando não o é, a variância assintótica e a taxa dde convergência do $E Q M I$ não são mais válidos.

Variáveis Aleatórias Dependentes. A maior parte dos resultados da estimação não-paramétrica de densidade foram estabelecidos para uma amostra i.i.d. Não obstante, alguns foram deduzidos sem a hipótese de independência, mas apenas para casos particulares: Roussas (1969, 1989, 1990), Rosenblatt (1991) Masry (1983) e Györfi et al (1989). Portanto, até recentemente, mais precisamente até o trabalho de Aït-Sahalia (1992), ao estimar-se densidades a partir de dados não independentes, era necessário calcular a distribuição limite do $E Q M I$ e sua taxa de convergência.

Ait-Sahalia (1992) provou um teorema central funcional do limite válido para qualquer funcional de um estimador de densidade. Enunciaremos apenas o Teorema 3-Colorário 2 desse trabalho, que estabelece a distribuição limite da densidade estimada univariada, sua variāncia assintótica e sua taxa de convergência. 


\section{Hipóteses}

(H1) A seqüência $\left\{X_{i}\right\}$ é uma seqüência $\beta$-mixing estritamente estacionária satisfazendo:

$$
k^{\delta} \beta_{k} \underset{k \rightarrow \infty}{\longrightarrow} 0 \quad \text { para dado } \delta>1
$$

Os coeficientes $\beta$-mixing $\left\{\beta_{i}\right\}$ são definidos como:

$$
\begin{gathered}
\beta_{k}=\sup _{I, J, 1}\left\{\sum_{i=1}^{I} \sum_{j=1}^{J}\left|P\left(A_{i} \cap B_{j}\right)-P\left(A_{i}\right) P\left(B_{j}\right)\right|\right. \\
\mid\left\{A_{i}\right\}_{i=1, \cdots, I} \text { seja uma partição de } \sigma_{t}^{1} \mathrm{e} \\
\left.\left\{B_{j}\right\}_{j=1, \cdots, I} \text { seja uma partição de } \sigma_{k+1}^{+\infty}\right\}
\end{gathered}
$$

onde $\sigma_{t}^{1}$ e $\sigma_{k+1}^{+\infty}$ são as $\sigma$-algebras geradas por $\left\{X_{1}, \cdots, X_{\ell}\right\}$ e $\left\{X_{t+1}, X_{t+1+1}, \cdots\right\}$ respectivamente.

Essa hipótese restringe o montante de dependência na amostra.

(H2) A função densidade acumulada (fda) $F(\cdot)$ é continuamente diferenciável em $R^{d}$ até a ordem $s+d$. A densidade $f(\cdot)$ tem suporte compacto $\Theta=[0,1]^{d}$ incluído em $R^{d}$, e suas derivadas são nulas no limite $\partial \Theta$ do suporte.

(H3) (i) $K$ é uma função para e $\int K(x) d x=1$, para todo $x \in R^{d}$.

(ii) Para $r=s$ :

1) $\forall \lambda \in N^{d}$ tal que $|\lambda| \equiv \lambda_{1}+\cdots+\lambda_{d} \in\{1, \cdots, r-1\}$

$$
\int_{-\infty}^{+\infty} X_{1}^{\lambda_{1}} \cdots X_{d}^{\lambda_{d}} K(x) d x=0
$$


2) $\exists \lambda \in N^{d l}$ tal que $|\lambda|=r$ e $\int_{-\infty}^{+\infty} X_{1}^{\lambda_{1}} \cdots X_{l l}^{\lambda_{d 1}} K(x) d x \neq 0$.

3) $\int_{-\infty}^{+\infty}\|x\|^{r}\left|K^{-}(x)\right| d x<+\infty$.

O núcleo $K(\cdot)$ satisfazendo (ii) é dito de ordem $r$..

(iii) $I f$ é continuamente diferenciável até a ordem $s+r$ em $R^{d}$, e suas derivadas de ordem até $s$ pertencem a $L_{2}\left(R^{d}\right)$. Estas serão designadas por $\mu(\Delta)$, para $\Delta \in N^{d}$ tal que $|\Delta| \equiv \Delta_{1}+\cdots+\Delta_{d}=q$ e $q=0,1, \cdots,(s-d)$ :

$$
\mu(\Delta) \equiv \partial^{(\Delta)} K(x) \equiv \frac{\partial^{|\Delta|} K}{\partial_{x_{1}}^{\Delta_{1}} \partial_{x_{2}}^{\Delta_{2}} \cdots \partial_{x_{d}}^{\Delta_{d}}}(x) .
$$

(H4) Para $e \in R$ e $m \in N, h_{n} \rightarrow 0$ quando $n \rightarrow \infty$ de modo que:

$$
n^{1 / 2} h_{n}^{e} \rightarrow 0 \quad \text { e } \quad n^{1 / 2} h_{n}^{2 m} \rightarrow+\infty .
$$

Essa hipótese indica como a janela $h_{n}$ deve ser escolhida. O resultado depende dos parâmetros $e>0$ e $0 \leq m \leq 2$ e que serão especificados no Teorema.

A função densidade estimada acumulada (fdea) é definida por $\hat{F}_{n}(x)=\int_{-\infty}^{+\infty} \hat{f}_{n}(t) d t$ e o processo estocástico $\hat{A}_{n}(x)$ é dado por $\hat{A}_{n}(x) \equiv n^{1 / 2}\left\{\hat{F}_{n}(x)-F(x)\right\}$. Sua convergência em distribuição será estabelecida pelo teorema abaixo. Para tanto, é necessário definir dois espaços de funções $C^{-1}$ e $C^{q}$.

$C^{-1}$ é o espaço de funções contínuas à direita e limitadas à esquerda de $[0,1]^{d}$ a $R$, e $C^{q}$ : para $0 \leq q \leq s$, o espaço de funçooes de $[0.1]^{d}$ a $R$ com derivadas contínuas até a ordem $q-s$. Ambos são dotados da norma do supremo.

Dadas essas definições, pode-se enunciar o seguinte: 
Teorema: Seja um funcional $\Phi[F] \equiv \partial^{(\Delta)} F(y)$ de um aberto $\overline{U^{s} \in C^{s}}$ a $R$. Suponha que seja diferenciável ${ }^{2}$ e que sua derivada funcional $\varphi[F]$ avaliada na verdadeira fda $F$ pertença a $C^{-q}$ para algum $q \in[2, s]$, então sbo as hipóteses $\mathrm{H} 1-\mathrm{H} 4$,

$$
h_{n}^{\left(2|\Delta|-l^{*}\right) / 2} n^{1 / 2}\left\{\Phi\left(\hat{F}_{n}\right)-\Phi(F)\right\} \stackrel{d}{\longrightarrow} N\left(0, V_{\Phi}(F)\right) .
$$

Nesse trabalho, estimamos a função densidade de probabilidade $f(x)=\partial^{(\Delta)} F(x)$, para $x \in \Re$, (portanto, $d=1$ ), para $\Delta=1=$ $(1, \cdots, 1)$, e $d^{*}=d$. De (iii) de H3 acima, tem-se $|\Delta|=1=q$. Por outro lado, o parâmetro $e=\left(r+d^{*}\right) / 2$ e $m$ é qualquer.

$\mathrm{O}$ resultado (16) pode portanto ser reescrito como:

$$
h_{n}^{1 / 2} n^{1 / 2}\left\{\hat{f}_{n}(y)-f(y)\right\} \stackrel{d}{\longrightarrow} N(0, \mu(0) f(y)
$$

onde $\mu(0)=\int_{-\infty}^{+\infty}|K(x)|^{2} d x .^{3}$

A taxa de convergência em (15) é $h_{n}^{\left(d^{*}+2 q-4\right) / 2} n^{1 / 2}$ e é sempre inferior a $n^{1 / 2}$ porque $h_{n} \rightarrow 0$ quando $n \rightarrow \infty$.

\section{Evidência Empírica.}

\subsection{Dados.}

As amostras analisadas fechamento de 4 ações do IBOVESPA. Paranapanema, Telebrás, Eletrobrás e Petrobrás, observadas durante o período 07/90 a 06/93. ${ }^{4}$ Essas cotações foram deflacionadas pelo IGP-DI centrado diário.

2 O conceito de diferenciabilidade desse funcional não é idêntico ao conceito de diferenciabilidade em $R^{n}$. P’ara maiores detalhes, ver Aït-Sahalia (199)2, seção II)

3 Ver a prova em Ait-Sahlalia (19)(2)

4 Tódos os preços ajustados para dividendos e bonificaçōes 


\section{Sylvia Delgado}

Estimou-se a função densidade de probabilidade da variação do logaritmo da cotação diária deflacionada:

$$
u_{t}=\ln p_{t}-\ln p_{t-1}
$$

que é o retorno da ação composto continuamente.

Como salientamos na introdução a esse trabalho, o objetivo não é determinar a forma funcional da distribuição de probabilidade do retorno dos preços das açَes, mas sim comparar o estimador déssa distribuição com a normal - em particular a $N(0,1)$. Para tanto, padronizamos a variável $u_{t}$ de forma a conseguir-se uma variável com média 0 e variäncia 1 .

Os estimadores que calculamos são baseados no núcleo "biweight" que possui a maior eficiência e deu resultados mais precisos que o gaussiano. As janelas foram escolhidas de forma a satisfazer a hipótese $\mathrm{H} 4$.

Os momentos da densidade estimada, que serão uma referência importante na comparação com a distribuição normal, foram calculados aproximando-se as integrais por somatórios:

$$
E[x]=\sum_{i} x_{i} \hat{f}\left(x_{i}\right)\left(x_{i+1}-x_{i}\right)
$$

por exemplo é a expressão do valor esperado. Lembramos que os coeficientes de assimetria e curtose da distribuição normal padrão são iguais a 0 e 3 respectivamente.

\subsection{Resultados.}

As funçøes densidade de probabilidade dos retornos das ações analisadas apresentam 3 características recorrentes nas distribuições de retornos de ativos financeiros: assimetria, leptocurtose e caudas mais pesadas do que as da distribuição normal. Isto implica que, comparando com a distribuição normal, (1) a probabilidade de 
grandes perdas e grandes ganhos é mais elevada e (2) a probabilidade de um retorno ern torno da média é também mais alta. Essas duas conseqüências são particularmente importantes na avaliação do risco de um investimento.

As discrepânciàs em relação à normalidade são maiores em algumas densidades estimadas do que em outras. A seguir apresentamos as principais propriedades de cada umi.

Paranapanema. A Figura 1 mostra a densidade estimada pelo estimador de núcleo dos retornos dessa ação.

Figuar 1:Densiddxde Estinekde I. C. a 95\%

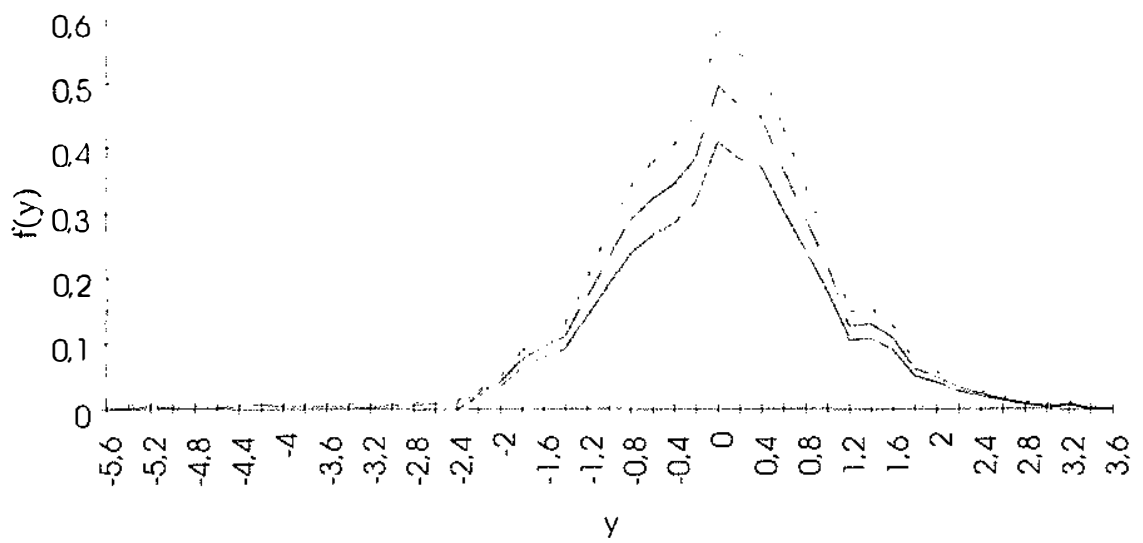


A assimetria à esquerda fica clara no gráfico e é confirmada pelo coeficiente de assimetria - calculado a partir da densidade estimada, que é igual a -0.39. A leptocurtose também é evidente. Por um lado, o coeficiente de curtose estimado é igual a 5,01 indicando uma distribuição mais pontuda que a distribuição normal, cujo coeficiente de curtose é 3,0 e, por outro lado, a probabilidade de um retorno entre 1 desvio-padrão em torno da média, ou seja, $P(-1<x<1)$, é igual a 0,77 na densidade estimada contra 0,68 na densidade normal.

Finalmente, a cauda esquerda da densidade estimada é muito mais pesada do que a da Normal, significando que o risco de um investimento em Paranapanema é mais elevado do que o risco de uma ação com retorno normalmente distribuído. Por exemplo, supondo-se o retorno da Paranapanema normalmente distribuído, ter-se-ia uma probabilidade de perda entre 4 e 5 desvio-padrão $99,7 \%$ inferior do que a probabilidade dessa mesma perda supondo-se os retornos distribuídos segundo a densidade estimada. E por outro lado, a probabilidade de um ganho entre 3 e 4 desvio-padrão é $67 \%$ mais elevada na densidade estimada do que na distribuição normal. Desse modo, o risco de uma perda/ganho é claramente subavaliado, se a distribuição dos retornos dessa ação forem considerados normais.

Petrobrás. A Figura 2 mostra uma densidade com as mesmas características da distribuição dos retornos da Paranapanema: assimetria à esquerda e leptocurtose. O coeficiente de assimetria é $-0,5$ e o de curtose 6.43.

Em relação às probabilidades de grandes perdas e grandes ganhos, observamos que estas são muito mais elcvadas do que as da distribuição normal: a probabilidade de uma perda entre 3 e 4 desviospadrão é $233 \%$ maior na densidade estimada e a probabilidade de uma perda entre 2 e 3 desvios-padrão ć 110\% maior. 
Figura 2:Densidade Estinxkhe I. C. a 95\%

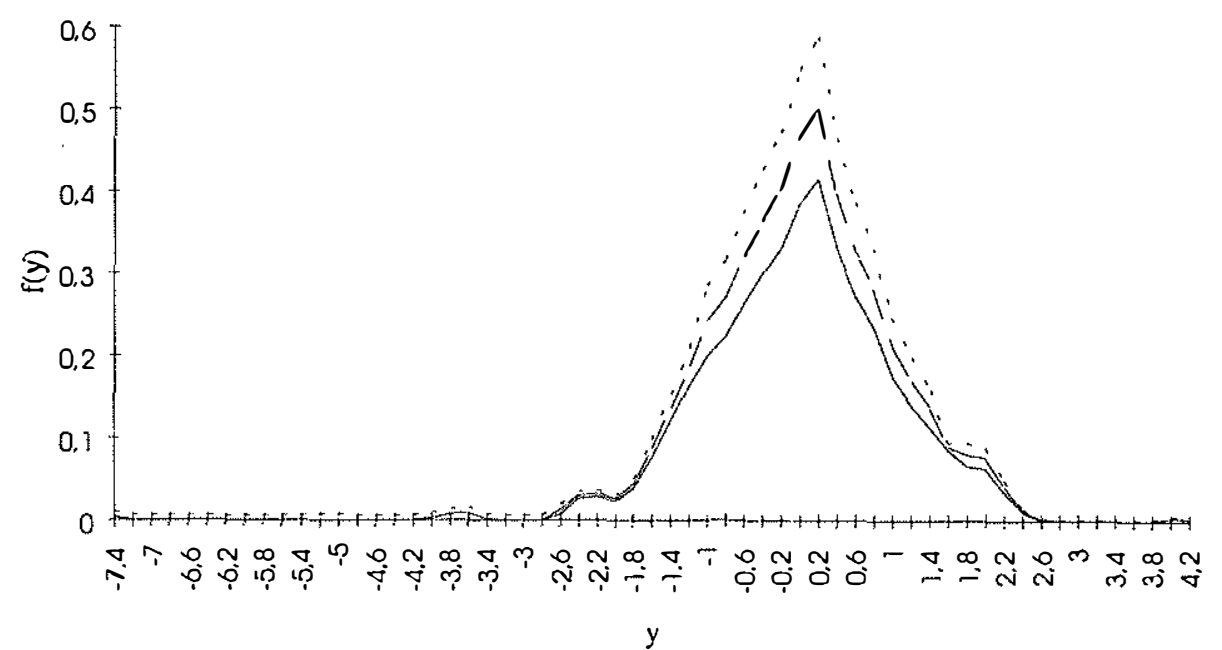

Para os retornos positivos, a diferença entre as probabilidades da normal e da densidade estimada são menos acentuadas: para um ganho entre 2 e 3 desvios-padrão, encontra-se uma probabilidade $31 \%$ maior na densidade estimada e entre 3 e 4 desvios-padrão a diferença é de $5 \%$. Por fim, a probabilidade de um retorno em torno da média, $P(-1<x<1)$, é igual 0,76 . Concluimos portanto que um investimento nessa ação é mais arriscado clo que $u$ im investimento numa ação com retornos normalmente distribuídos, e que a probabilidade de retorno médio é mais elevada.

Telebrás. Das 4 ações analisadas, esta é a úniča com retornos praticamente simétricos e com caudas menos pesadas do que as da distribuição normal, como pode ser visto na Figura 3 . O coeficiente de assimetria e curtose estimados são iguais a $-(0.14$ e 3,85, indicando 
uma densidade ligeiramente assimétrica à esquerda e leptocúrtica.

Figura 3: Densidade Estimedn c I. C. a $95 \%$

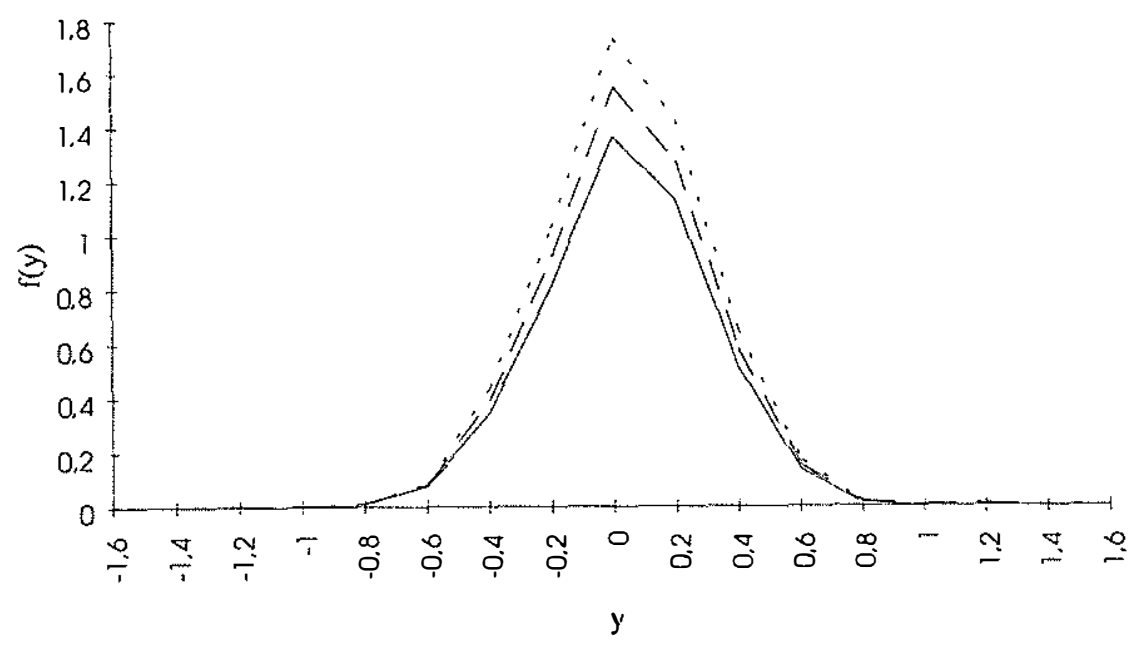

A distribuição dos retornos da Telebrás é fortemente concentrada em torno da média, com 99,8\% de sua área entre 1 desvio-padrão cm torno da média, ou seja, a probabilidade de um retorno em torno da média é de praticamente $100 \%$.

Como conseqüencia, a probabilidade de perda e de ganho superior a 2 desvios-j)adrão pode ser considerada nula, o que implica um risco de perda/ganho elevados nuito baixa. Entre 0,5 e 1,5 desviopadrāo, a probabilidade de perda (ganho) é $959 \%$ (516\%) mais elevada na distribuição normal. Isso nos leva a concluir que supor os retornos da Tclebrás normalmente distribuídos implica numa superavaliação do risco de um investimento nessa ação. 
Eletrobrás. Finalmente, a Figura 4 mostram os estimadores de densidade da Eletrobrás. Ambos evidenciam uma densidade assimétrica à esquerda e leptocúrtica. A curva do estimador de núcleo é bastante acidentada apresentando urna moda no intervalo $[-2,4 ;-1,2]$. A área sob a curva no intervalo $[-5,2 ; 0]$ representa $65 \%$ da área total, confirmando a assimetria à esquerda.

\section{Figura 4:Densidexde Estinnda e I.C. a 95\%}

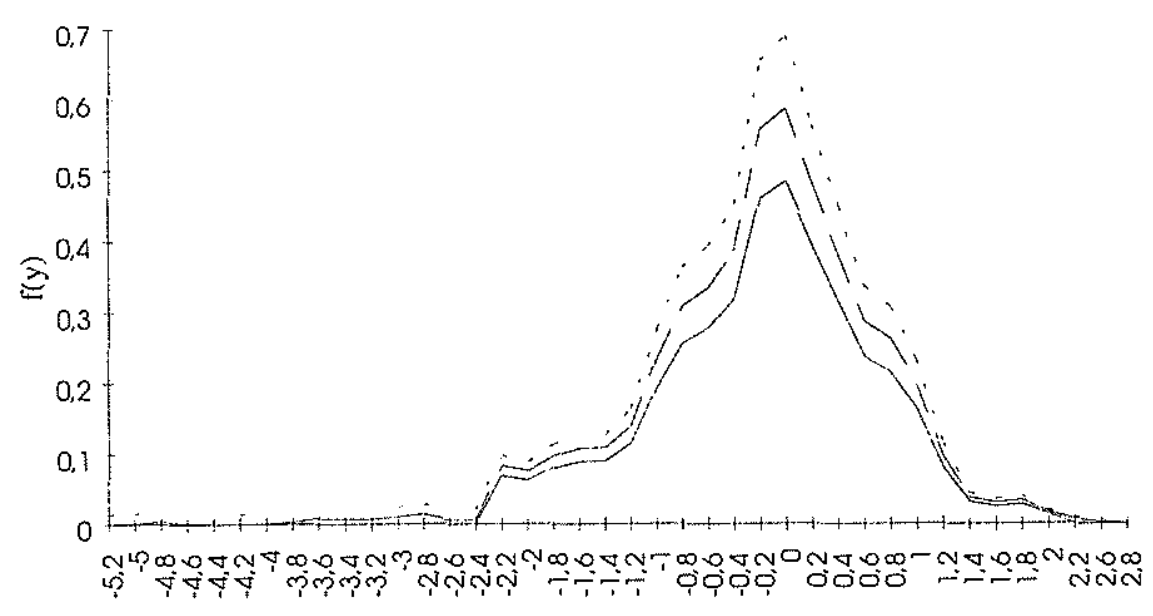

Em relação à leptocurtose, not,amos que seu coeficiente de curtose é igual a 4,62 e que $81 \%$ da área tot,al se encontra entre 1 desviopadrão cm torno da míclia, ou seja, a probabilidade de um retorno em torno da média é mais elevada do que essa mesma probabilidade supondo-se a normalidade.

A cauda esquerda da distribuição estimada é mais pesada e a direita menos pesada do que as da normal: a probabilidade de uma perda acima de 4 desvios-padrão ć $4.330 \%$ mais elevada na densidade 


\section{Sylvia Delgado}

estimada! e as probabilidades de perda entre 3 e 4 , e 2 e 3 desviospadrão são ambas $81 \%$ inferiores na distribuição normal.

O comportamento da distribuição de probabilidade dos retornos da Eletrobrás é semelhante ao dos retornos da Petrobrás e Paranapanema, logo, o risco de um investimento nessa ação e a probabilidade de ganho em torno da média são mais elevados do que o risco e a probabilidade de ganho de um investimento numa ação com retornos normalmente distribuídos.

\section{Testes de Normalidade.}

A título de comparação, efetuamos 3 testes de normalidade sobre os retornos padronizados das ações Paranapanema, Petrobrás e Eletrobrás. Esses testes são o Qui-Quadrado, o Kolmogorov-Smirnov e o teste de Jarque-Bera (J-B).

As seguintes tabelas resumen os resultados dos 3 testes de normalidade que efetuamos:

\section{Tabela 1: Paranapanema}

\begin{tabular}{|c|r|c|}
\hline Teste & Valor do Teste & p-valor \\
\hline Qui-quadrado & 20,66 & 0,08 \\
IK-S & 0,0394 & 0,21 \\
J-B & 49,61 & 0,00 \\
\hline
\end{tabular}

Tabela 2: Telebrás

\begin{tabular}{|c|r|c|}
\hline Teste & Valor do Teste & p-valor \\
\hline Qui-quadrado & 16,09 & 0,0097 \\
K-S & 0,04 & 0,13 \\
J-B & 73,29 & 0,00 \\
\hline
\end{tabular}


Retornos das ações no Brasil

Tabela 3: Eletrobrás

\begin{tabular}{|c|c|c|}
\hline Teste & Valor do Teste & p-valor \\
\hline Qui-quadrado & 40,68 & 0,00 \\
K-S & 0,071 & 0,01 \\
J-B & 2956 & 0,00 \\
\hline
\end{tabular}

Tabela 4: Petrobrás

\begin{tabular}{|c|c|c|}
\hline Teste & Valor do Teste & p-valor \\
\hline Qui-quadrado & 20,23 & 0,0 \\
K-S & 0,032 & 0,47 \\
J-B & 132,1 & 0,00 \\
\hline
\end{tabular}

O teste Kolmogorov-Smirnov aceita a hipótese de normalidade dos retornos da Petrobrás, Telebrás e Paranapanema ao nível de 5\% e a aceita no caso da Eletrobrás ao nível de $1 \%$.

O teste Chi-quadrado rejeita a hipótese de normalidade dos retornos da Petrobrás, Eletrobrás e Telebrás a qualquer nível de significância e a aceita no caso da Paranapanema a 5\%.

$\mathrm{O}$ teste $\mathrm{J}$-B rejeita a hipótese nula em todos os casos, a $5 \%$ e $1 \%$.

Em resumo, pode-se aceitar que os retornos da Eletrobrás, Telebrás, Petrobrás e Paranapanema não são normalmente distribuídos, confirmando as conclusões da estimação não-paramétrica.

\section{Conclusão.}

Todas as distribuições de probabilidade estimadas apresentam características que as diferenciam da distribuição normal: forte assimetria, leptocurtose com uma das caudas mais pesada nas densidades dos retornos da Paranapanema, Petrobrás e Eletrobrás; leptocurtose com ambas caudas menos pesadas no caso da Telebrás. 
Em termos de investimento, isso significa que todas as ações que estudamos aqui têm uma probabilidade de retorno em torno da média superior a essa mesma probabilidade supondo-se a distribuição normal. Essa afirmação nãoé preocupante para um investidor racional, visto que seu objetivo ao investir é o retorno médio. Por outro lado, a assimetria implica uma maior probabilidade de perda - ou ganho dependendo do tipo de assimetria. Nos nossos casos, sempre verificamos uma assimetria à esquerda. Isto significa que a probabilidade de uma perda é mais elevada supondo-se que os retornos seguem a distribuição estimada do que se eles fossem normalmente distribuídos.

Dois motivos foram apontados para explicar a existência de caudas pesadas nas distribuições de retornos: primeiro, essa distribuição pode resultar da mistura de duas ou mais distribuições normais com variãncias e médias diferentes, ou a mistura de distribuições normais e log-normais. A pesquisa realizada nesse sentido por Clark (1973) e Praetz (1972), por exemplo, não foi conclusiva, devido à dificuldade de se estimar os parãmetros desse tipo de distribuição, mas admite-se que a mescla uma normal e uma log-normal seja uma boa aproximação. Sugere-se também que a densidade dos retornos das ações seja uma $t$ de Student, que se caracteriza justamente por suas caudas mais pesadas e apresenta a vantagem de convergir em distribuição para a densidade normal. Vários testes comparando os modelos normal, $t$ de Student e Pareto concluíram a favor de $t$ de Student. O trabalho de Blattberg e Gonedes (1964) constitui uma excelente referência sobre o assunto, visto que resumem toda a pesquisa sobre processos estocásticos e testes de normalidade efetuados até 1974 . Além disso, desenvolvem um bom método de comparação entre o modelo Pareto e $t$ de Student.

O segundo motivo pode ser que a variância dos retornos de ações seja finita mas não constante ao longo do tempo. Nesse caso, os retornos viriam de processos do tipo GARCH. De fato, a atual pesquisa empírica sobre ativos financeiros indica que os modelos ARCH-GARCH são particularmente adequados para explicar o com- 
portamento desses ativos. No Brasil, por exemplo, Fernandes (1994) mostrou que a volatilidade do IBV-RJ podia ser perfeitamente modelada como um GARCH.

Submetido em Novembro de 1994. Revisado em Julho de 1995.

\section{Referências}

Ait-Sahalia, Y. 1992. "The delta and bootstrap methods for nonlinear functionals of nonparametric kernel estimators based on dependent multivariate data". Mit, Mimeo, Janeiro.

Bickel, P.J. \& Doksum, K.A. 1977. Mathematical Statistics. HoldenDay Inc.

Blattberg, R.C. \& Gonedes, N.J. 1974. "A comparison of the stable and student distributions as statistical models for stock prices". Journal of Business. 47: 244-280.

Clark, P.K. 1973. "A subordinated stochastic process model with finite variance for speculative prices". Econometrica. 41: 135155.

Durbin, J. 1973. "Distribution theory for tests based on the sample distribution function". SIAM: Regional Conference Series in Applied. Math. 9, Phil., Penn.

Fama, E. 1965. "The behavior of speculative prices". Journal of Business. 38: 34-105.

Fernandes, M. 1994. "Volatilidade da bolsa cle valores do Rio cle Janeiro". Anais do XVI Encontro Brasileiro de Econometria, Dezembro.

Gasser, T.; Muller, H-G. \& Mammitzsch, V. 1985. "Kernels for nonparametric curve estimation". Journal of the Royal Statistical Society. 47, Ser. B: 238--252. 
Györfi, L.; Härdle, W.; Sarda, P. \& Vieu, P. 1989. "Nonparametric curve estimation from time series", Lecture Notes in Statistics. 60, Springer-Verlag.

Izenmann, A.J. 1991. "Recent developments in nonparametric density estimation". Journal of the American Statistical Assocation, 86: 205--224.

Johnson, N. \& Kotz, S. 1970. Distributions in Statistics: Continuous Univariate Distributions. Houghton Mifflin Company.

Mandelbrot, B. 1963. "The variation of certain speculative prices". Journal of Business. 36: 349-419.

Masry, E. 1983. "Probability density estimation from sampled data". IEEE Iransactions on Information Theory. IT-29: 696709.

Moore, A.B. 1965. Some Characteristics of Changes in Common Stock Prices. In Cootner 1964.

Osborne, M.F.M. 1959. "Brownian motion in the stock market". Operational Research. 7: 145-173.

Parzen, E. 1962. "On estimation of a probability density function and mode". The Annals of Mathematical Statistics. 33: 10651076.

Perry, P.R. 1983. "More evidence on the nature of the distribution of security returns". Journal of Financial and Quantitative Analysis. 18: 211-221.

Praetz, P.D. 1972. "The distribution of share price changes". Journal of Business. 45: 49-55.

Rosenblatt, M. 1956. "Remarks on some nonparametric estimates of a density function". The Annals of Mathematical Statistics. 27: 832-837.

. 1991. "Stochastic curve estimation". NSF-CMBS Regional Conference Series in Probability and Statistics. Hay ward: Institute of Mathematical Statistics. 
Roussas, G. 1969. "Nonparametric estimation in Markov processes". Annals of the Institute of Statistical Mathematics. 21: 832-835. . 1989. "Hazard rate estimation under dependence conditions". Journal of Statistical Planning and Inference. 22: 81-93. . 1990. "Asymptotic normality of the kernel estimate under dependence conditions: application to Hazard rate". Journal of Statistical Planning and Inference. 25: 81-93.

Shapiro, S.S.; Wilk, M.B. \& C'hen, H.J. 1986. "A comparative study of various tests of normality". American Statistical Society Journal. December. 